Research Article

\title{
Researches on Damage Evolution and Acoustic Emission Characteristics of Rocks
}

\author{
Yong Chen $\mathbb{D}^{1},{ }^{1}$ Yugui Yang $\mathbb{D}^{2},{ }^{2}$ Feng Gao, ${ }^{2}$ and Xiangxiang Zhang ${ }^{2}$ \\ ${ }^{1}$ State Key Laboratory of Coal Resource and Safe Mining, China University of Mining and Technology, Xuzhou, \\ Jiangsu 221116, China \\ ${ }^{2}$ State Key Laboratory for Geomechanics and Deep Underground Engineering, China University of Mining and Technology, \\ Xuzhou, Jiangsu 221008, China \\ Correspondence should be addressed to Yugui Yang; ygyang2009@126.com
}

Received 6 September 2017; Revised 11 December 2017; Accepted 8 January 2018; Published 2 April 2018

Academic Editor: Rihong Cao

Copyright ( $\odot 2018$ Yong Chen et al. This is an open access article distributed under the Creative Commons Attribution License, which permits unrestricted use, distribution, and reproduction in any medium, provided the original work is properly cited.

\begin{abstract}
Mechanical parameters of the rock are important for the design of geotechnical, mining engineering, and petroleum reservoir projects. Many researches have suggested that the mechanical variables of rock specimens, such as compressive strength and elastic modulus, do not have a single fixed value. Uncertainty in the basic mechanical variables of the rock material can significantly affect the structural performance and safety. In this study, a series of compression experiments with acoustic emission have been performed on rock specimens. The damage evolution characteristics of the rock in the process of loading were studied, and the macromechanical behaviors were obtained at the same time. Distribution characteristics of the strength and elastic modulus as random variables are illustrated, and the statistical damage model is presented by the authors to formulate analytical constitutive relations for deformation behavior. The comparisons between predicted results and experimental data show that the statistical damage constitutive model could well reproduce the deformation process of rock materials.
\end{abstract}

\section{Introduction}

Mechanical property of the rock is important for the design of geotechnical, mining engineering, and petroleum reservoir projects. A large number of experiments suggested that the mechanical variables of rock specimens, such as compressive strength and elastic modulus, do not have a single fixed value [1-4]. The measured mechanical parameters for a given rock usually show a large scatter characteristic. There is no way of predicting exactly what the values of these parameters are. Uncertainty in the basic mechanical variables of the rock material can significantly affect the structural performance and safety. Understanding this variation, or uncertainty, in the design and analysis of geotechnical engineering requires the use of structural reliability-based design and assessment methodologies [5]. Specimens with the same nominal material parameters and tested under the same environmental conditions may exhibit different behaviors with diversified strength and should be analyzed by a distribution function [6]. Hence, regarding these mechanical parameters as random variables, the statistical mechanical approach, which has been applied successfully to deal with the mechanical behavior of the rock, becomes a quite attractive tool for investigating deformation processes and failure mechanism of the geomaterial [7-9]. In fact, the diversity of mechanical behavior for specific samples is usually attributed to the heterogeneity of the material at the mesoscopic level. The most important aspect of the engineering material is its microstructure, which contains many randomly distributed microcracks. The rock material is initially statistically homogeneous but becomes heterogeneous due to the propagation and clustering of microcracks [10]. However, the effect of mesoscopic heterogeneity on macroscopic failure is still not clear. Furthermore, considering that mechanical variables of the rock material exhibit great uncertainty, it is unreasonable to take some deterministic values as the mechanic parameters, and the reliability analysis of the mechanical variable for the rock material is necessary. 


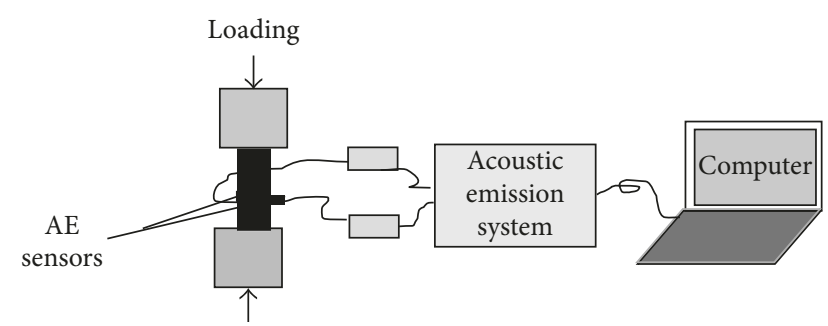

Figure 1: Collected and analyzed system of the acoustic emission.

In this paper, a series of acoustic emission (AE) experiments with uniaxial compression have been performed, and the macromechanic properties and microdamage evolution have been investigated. Distributions of the strength and elastic modulus as random variables are illustrated by several samples of rock specimens, respectively. The normal, logarithmic-normal, and Weibull distributions are used to describe the statistical characteristics. The damage mechanics is introduced to deal with the deformation characteristic of sandstone.

\section{Experiments}

The tests were performed with MTS815, and the acoustic emission testing system was illustrated in Figure 1. The sandstone specimens were prepared with the dimension of the cylindrical specimen $50 \times 100 \mathrm{~mm}$. The AE detection model PCI- 2 of the DISP series manufactured by PAC was employed to detect $\mathrm{AE}$ signals from the specimens. The $\mathrm{AE}$ sensors were attached to the outer wall of the rock specimen for good signal detection. Data logging was controlled by a computer during testing. After the specimen was placed on the fixed lower head of the testing machine, the compressive load was applied to the specimens until failure with the strain rate of $1.5 \times 10^{-5} / \mathrm{s}$. The upper moving head was brought down until it just touched the specimen.

The stress-strain curves of rocks are shown in Figure 2. From Figure 2, it can be seen that the shapes of stress-strain curves are similar in the process of loading. The stress-strain curve can be approximately divided into four typical stages: (1) the compaction stage: the stress-strain curve of the sample shows the downward concave and the initial nonlinear deformation at low stress levels. At the initial process of loading, the pores, voids, and microcracks of the rock sample are compacted with the increase of compression strain; (2) the linear elastic stage: after the initial compaction, stress increases approximately linearly with the further increase of axial strain, and there is little residual strain in this stage; (3) the crack propagation stage: at this stage, the slope of the stress-strain curve gradually decreases with the further increase of axial strain, the evolution of damage during compression loading causes a small amount of residual deformation, and the rigidity of the rock sample reduces; (4) the failure stage: when the microcrack develops to a certain extent, the macroscopic crack in the rock sample comes out rapidly and the stress-strain curves shows a rapid drop in the postpeak region, and the failure behavior of the rock presents an obvious brittle failure characteristic under

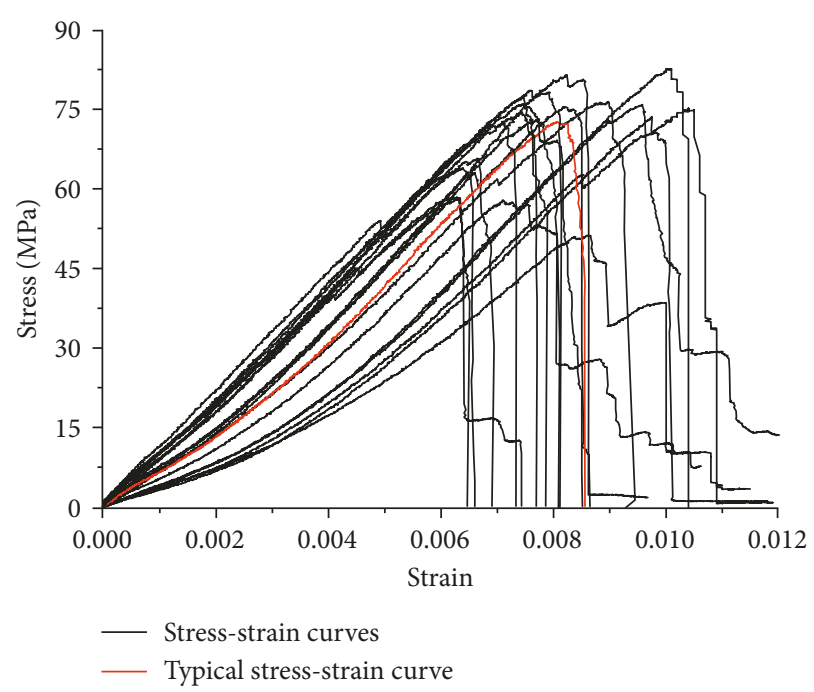

FIgURE 2: The stress-strain curves of sandstone under uniaxial compression.

uniaxial compression conditions. From Figure 2, it can also be seen that the stress-strain curves are significantly different from each other under the same compression conditions. Specimens with the same nominal material parameters and environmental conditions exhibit different behaviors with diversified strength and should be analyzed by a distribution function. It is necessary to adopt the probabilistic approach to study the mechanical behaviors, which takes the discreteness of mechanical parameters into account.

Acoustic emission data were analyzed to describe the damage evolution. These correlations are illustrated in Figure 3. Test results show that the rock emits a small number of $\mathrm{AE}$ events before entering the failure stage. AE activity gradually increases with the increase of strain. It is shown that this $\mathrm{AE}$ parameter is a reasonable indicator for damage occurring within the rock specimen, except for a short time delay. The stress and strain lag behind $\mathrm{AE}$ activity. In the compaction stage, a few events appear suggesting that little damage of the rock happens. The elastic stage, corresponding to a linear variation of strain with stress, exhibits a slow increase of $\mathrm{AE}$ activity. As the strain increases to a critical value, the $\mathrm{AE}$ activity increases rapidly [11]. The regularities of AE activities are different in the loading process for different failure types. The strenuous activity interval of the AE activities is narrow in the failure stage under the condition of tensile failure; for the shear failure, the strenuous activity interval of the AE activities becomes wide.

\section{Probability Distribution Characteristics of the Rock Material}

The variations of mechanical parameters of rocks are very large; it is unsafe and unreasonable for engineering design to use the average strength. In this study, the reliability of the mechanical variables, such as strength and elastic modulus, was investigated and discussed. The cumulative probabilistic distribution of data with $N$ number of samples was calculated 


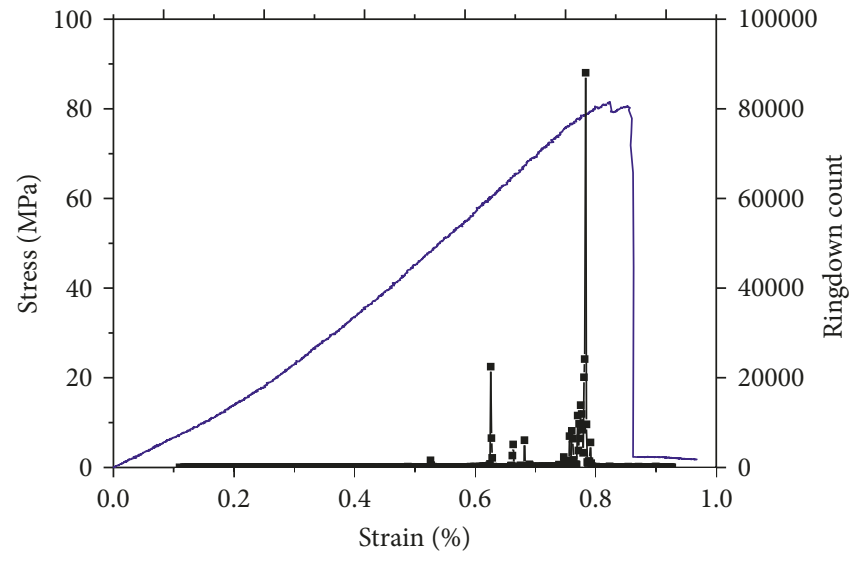

(a)

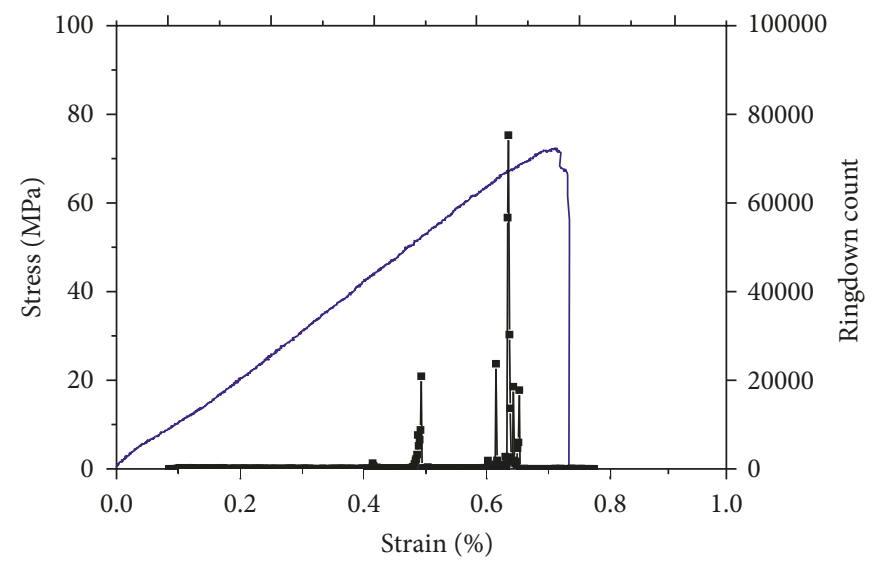

(b)

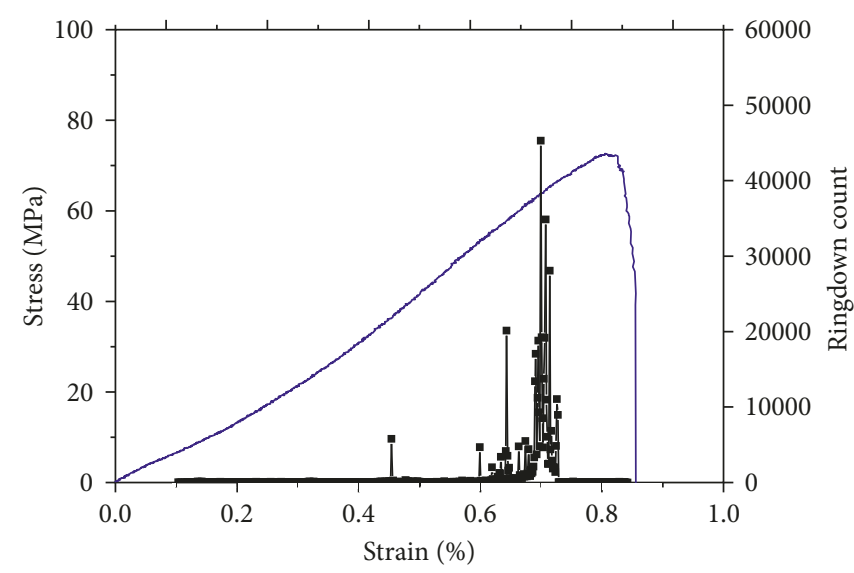

(c)

FIgURE 3: Acoustic emission characteristics for different failure types: (a) acoustic emission for tensile failure, (b) acoustic emission for combination failure, and (c) acoustic emission for shear failure.

by ranking the data from the lowest value to the highest value using (1). The probability of the specimen is given by [12]

$$
P_{f}^{i}=\frac{i-0.5}{N}
$$

where $i$ is the ranking of the data.

As it is customary for brittle materials, the strength data are interpreted statistically by assuming that specimen strength obeys a probability density function. In this section, we briefly describe different probabilistic models considered here and mention the estimation procedures of the unknown parameters from a given sample dataset. The performance of the models was then compared by matching the calibrated models with the individual test results.

3.1. Normal Distribution. The probability density function of a normal distribution is given as follows [13]:

$$
f(x)=\frac{1}{\sqrt{2 \pi} \sigma} e^{(x-\mu)^{2} / 2 \sigma^{2}},
$$

where $x$ is the value of the continuous random variable.

The cumulative distribution function for the normal distribution is given as follows:

$$
F(x)=\frac{1}{\sqrt{2 \pi} \sigma} \int_{-\infty}^{x} e^{(x-\mu)^{2} / 2 \sigma^{2}} d t
$$

Maximum-likelihood estimation is a method of estimating the parameters of a statistical model. When applied to a dataset and given a statistical model, maximumlikelihood estimation provides estimates for the model's parameters. For normal distribution, the likelihood for the whole sample is given as follows:

$$
\begin{aligned}
L & =\prod_{i=1}^{n} \frac{1}{\sqrt{2 \pi} \sigma} e^{-\left(\left(x_{i}-\mu\right)^{2} / 2 \sigma^{2}\right)} \\
& =\left(\frac{1}{\sqrt{2 \pi}}\right)^{n}\left(\frac{1}{\sigma^{2}}\right)^{n / 2} e^{-\left(1 / 2 \sigma^{2}\right) \sum_{i=1}^{n}\left(x_{i}-\mu\right)^{2}} .
\end{aligned}
$$

Taking log, the log-likelihood is given as follows:

$$
\ln L=n \ln \left(\frac{1}{\sqrt{2 \pi}}\right)-\frac{n}{2} \ln \sigma^{2}-\frac{1}{2 \sigma^{2}} \sum_{i=1}^{n}\left(x_{i}-\mu\right)^{2} \text {. }
$$

To find the critical points of the log-likelihood function, set the first derivative with respect to each equal to zero. In differentiating (5), note that 


$$
\begin{aligned}
& \frac{\partial \ln L}{\partial \mu}=\frac{1}{\sigma^{2}} \sum_{i=1}^{n}\left(x_{i}-\mu\right), \\
& \frac{\partial \ln L}{\partial \sigma^{2}}=-\frac{n}{2 \sigma^{2}}+\frac{1}{2 \sigma^{4}} \sum_{i=1}^{n}\left(x_{i}-\mu\right)^{2} .
\end{aligned}
$$

The maximum-likelihood estimates for $\mu$ and $\sigma$ can be found by setting (6) and (7) equal to zero, and solving for each, $\mu$ and $\sigma$ can be obtained as follows:

$$
\begin{aligned}
\mu & =\frac{1}{n} \sum_{i=1}^{n} x_{i}, \\
\sigma^{2} & =\frac{1}{n} \sum_{i=1}^{n}\left(x_{i}-\mu\right)^{2} .
\end{aligned}
$$

3.2. Logarithmic-Normal Distribution. In probability theory, a log-normal distribution is a continuous probability distribution of a random variable whose logarithm is normally distributed. The probability density function of a log-normal distribution is given as follows [14]:

$$
f(x)= \begin{cases}\frac{1}{\sqrt{2 \pi} \sigma x} e^{-\left((\ln x-\mu)^{2} / 2 \sigma^{2}\right)} & (x>0) \\ 0 & (x \leq 0) .\end{cases}
$$

The cumulative distribution function for the normal distribution is given as follows:

$$
F(x)=\frac{1}{\sqrt{2 \pi} \sigma} \int_{-\infty}^{x} e^{-\left((\ln t-\mu)^{2} / 2 \sigma^{2}\right)} \frac{1}{t} \mathrm{dt} .
$$

For determining the maximum-likelihood estimators of the log-normal distribution parameters $\mu$ and $\sigma$, we can use the same procedure as used in the normal distribution. Therefore, using the same indices to denote distributions, we can write the log-likelihood function as follows:

$$
\begin{aligned}
L & =\prod_{i=1}^{n} \frac{1}{\sqrt{2 \pi} \sigma x_{i}} e^{-\left(\left(\ln x_{i}-\mu\right)^{2} / 2 \sigma^{2}\right)} \\
& =\left(\frac{1}{\sqrt{2 \pi}}\right)^{n}\left(\frac{1}{\sigma^{2}}\right)^{n / 2} e^{-\left(1 / 2 \sigma^{2}\right) \sum_{i=1}^{n}\left(x_{i}-\mu\right)^{2}}
\end{aligned}
$$

Now taking log, the log likelihood is obtained as follows:

$\ln L=-n \ln (\sqrt{2 \pi} \sigma)-\sum_{i=1}^{n} \ln x_{i}-\sum_{i=1}^{n} \frac{\left(\ln x_{i}-\mu\right)^{2}}{2 \sigma^{2}}$.

By differentiating (12), we have

$$
\begin{aligned}
& \frac{\partial \ln L}{\partial \mu}=\frac{1}{\sigma^{2}} \sum_{i=1}^{n}\left(\ln x_{i}-\mu\right) \\
& \frac{\partial \ln L}{\partial \sigma^{2}}=-\frac{n}{2 \sigma^{2}}+\frac{1}{2 \sigma^{4}} \sum_{i=1}^{n}\left(\ln x_{i}-\mu\right)^{2} .
\end{aligned}
$$

Using the formulas of the normal distribution maximum-likelihood parameter method, we deduce that, for the log-normal distribution, it holds that

$$
\begin{aligned}
\mu & =\frac{1}{n} \sum_{i=1}^{n} \ln x_{i}, \\
\sigma^{2} & =\frac{1}{n} \sum_{i=1}^{n}\left(\ln x_{i}-\mu\right)^{2} .
\end{aligned}
$$

3.3. Weibull Distribution. The Weibull distribution is one of the most widely used lifetime distributions in reliability engineering. It is a versatile distribution that can take on the characteristics of other types of distributions. The probability density function of the Weibull distribution is given as follows [15]:

$$
f(x)= \begin{cases}\frac{k}{\lambda}\left(\frac{x-\theta}{\lambda}\right)^{k-1} e^{-(x-\theta / \lambda)^{k}} & (x \geq 0) \\ 0 & (x<0),\end{cases}
$$

where $k$ is the shape parameter, $\lambda$ is the scale parameter of the distribution, and $\theta$ is the location parameter of the distribution.

The cumulative distribution function for the Weibull distribution is

$$
F(x)=1-e^{-(x-\theta / \lambda)^{k}}
$$

Considering the Weibull distribution given in (15), the likelihood function will be

$$
L=\prod_{i=1}^{n} f\left(x_{i}\right)=\prod_{i=1}^{n} \frac{k}{\lambda}\left(\frac{x_{i}-\theta}{\lambda}\right)^{k-1} e^{-\left(x_{i} / \lambda\right)^{k}} .
$$

On taking the logarithms of (17), we obtain the estimating equation as follows:

$$
\begin{gathered}
\ln L=\prod_{i=1}^{n} f\left(x_{i}\right) \\
=n \ln \frac{k}{\lambda}+(k-1) \sum_{i=1}^{n} \ln \left(\frac{x_{i}-\theta}{\lambda}\right)-\frac{1}{\lambda^{k}} \sum_{i=1}^{n}\left(x_{i}-\theta\right)^{k} .
\end{gathered}
$$

In differentiating (18) with respect to $\lambda, k$, and $\theta$ in turn, we have

$$
\begin{aligned}
& \frac{\partial \ln L}{\partial \lambda}=-\frac{n k}{\lambda}+\frac{k}{\lambda^{k+1}} \sum_{i=1}^{n}\left(x_{i}-\theta\right)^{k} \\
& \frac{\partial \ln L}{\partial k}=-\frac{n}{k}+\sum_{i=1}^{n} \ln \left(\frac{x_{i}-\theta}{\lambda}\right)-\sum_{i=1}^{n}\left(\frac{x_{i}-\theta}{\lambda}\right)^{k} \ln \left(\frac{x_{i}-\theta}{\lambda}\right)^{k}, \\
& \frac{\partial \ln L}{\partial \theta}=(1-k) \sum_{i=1}^{n}\left(x_{i}-\theta\right)^{-1}+\frac{k}{\lambda} \sum_{i=1}^{n}\left(\frac{x_{i}-\theta}{\lambda}\right)^{k-1} .
\end{aligned}
$$

The parameters $\lambda, k$, and $\theta$ can be accomplished by the use of iterative procedures. Figures 4 and 5 show the theoretical distribution relationships together with the test results for comparison. It can be seen that the normal and 


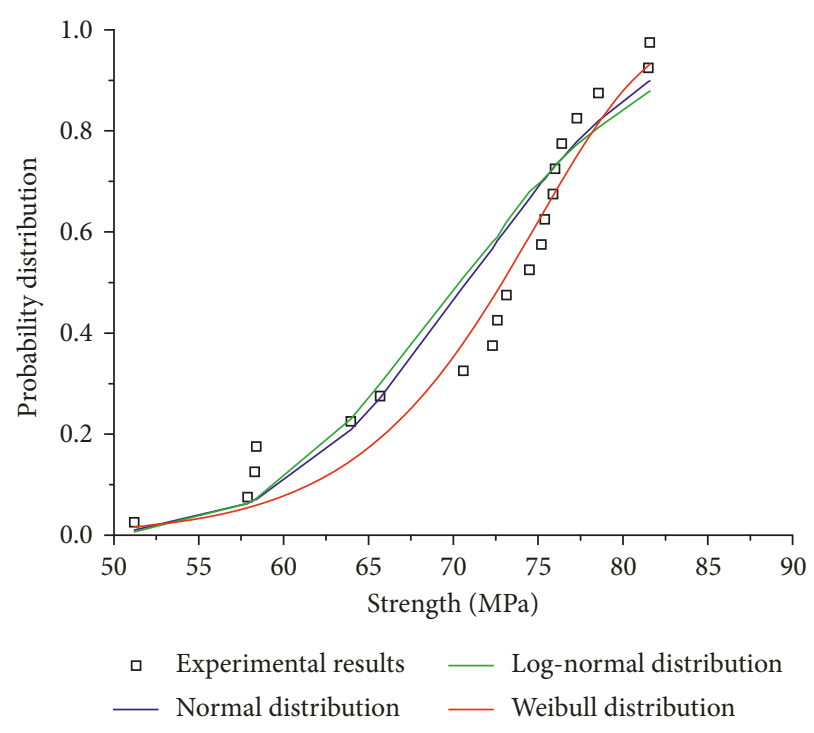

FIgURe 4: Probability distribution characteristic of the strength under compression.

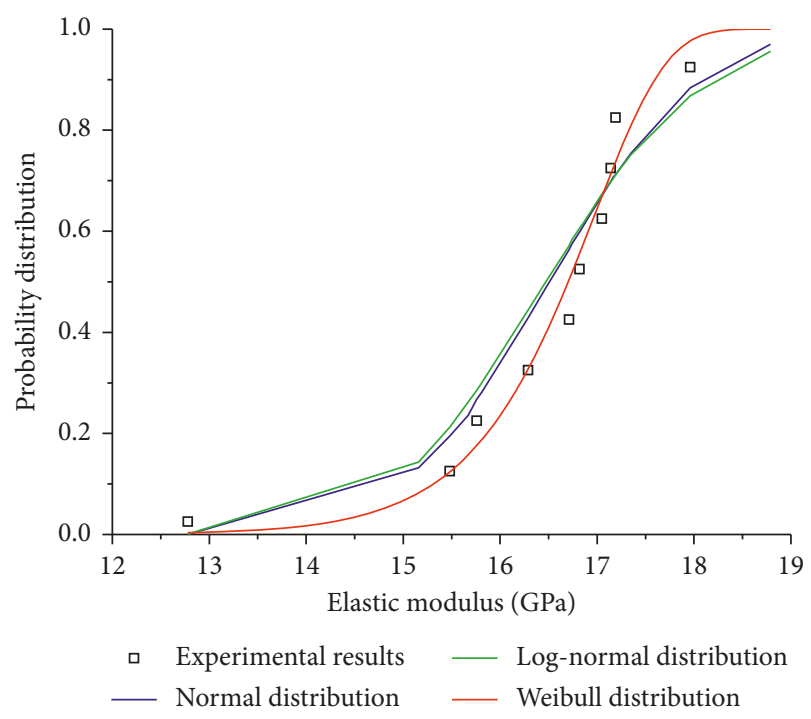

Figure 5: Probability distribution characteristic of the elastic modulus under compression.

logarithmic-normal distribution models are very similar, but neither of these models matches with the test results better than the Weibull distribution model. To analyze exactly the precision of the three kinds of probability distributions, the maximum difference between the experimental and theoretical data for the three distributions is presented in Table 1. From Table 1, it can be seen that the maximum differences of the strength between the theoretical results and experimental data are different, and their values are $0.192,0.146$, and 0.116 , respectively. The maximum differences of the elastic modulus are $0.138,0.146$, and 0.053 , respectively. The Weibull distribution has less difference between the experimental data and theoretical results either in strength or elastic modules. It can be
TABLE 1: Maximum differences between experimental and theoretical distributions.

\begin{tabular}{lcc}
\hline Probability distribution & Elastic modulus & Strength \\
\hline Normal distribution & 0.138 & 0.192 \\
Logarithmic-normal distribution & 0.146 & 0.205 \\
Weibull distribution & 0.053 & 0.116 \\
\hline
\end{tabular}

TABLE 2: Confidence levels for the strength and elastic modulus of sandstone.

\begin{tabular}{lcc}
\hline Confidence & $\begin{array}{c}\text { Elastic modulus } \\
(\mathrm{GPa})\end{array}$ & $\begin{array}{c}\text { Strength } \\
(\mathrm{MPa})\end{array}$ \\
\hline 0.90 & $15.32+\infty$ & $61.50+\infty$ \\
0.95 & $14.82+\infty$ & $57.38+\infty$ \\
0.99 & $13.59+\infty$ & $51.20+\infty$ \\
Normal distribution & 0.138 & 0.192 \\
Logarithmic-normal distribution & 0.146 & 0.205 \\
Weibull distribution & 0.053 & 0.116 \\
\hline
\end{tabular}

concluded that the probability distribution law is satisfied slightly better by the Weibull distribution rather than the other distributions.

In order to ensure safety and save cost in engineering construction, the choice of strength design is important. In statistics, a confidence interval is a type of an interval estimate of the population parameter and is used to indicate the reliability of an estimate. The confidence levels for the sandstone are listed in Table 2. It can be seen that when the uniaxial compressive strength is taken as $61.50 \mathrm{MPa}$, the safe reliability is $90 \%$, and when the strength is taken as $57.38 \mathrm{MPa}$, the undamaged probability is $95 \%$. A confidence level of $95 \%$ means that there is a probability of at least $95 \%$ that the result is reliable.

\section{Damage Constitutive Model of Rocks}

So far, a variety of constitutive models for brittle or ductile materials have also been developed and implemented within the damage mechanic framework incorporating statistical variations [16-18]. The statistical damage model presented by the authors in the previous article is used to formulate analytical constitutive relations for deformation behavior. Based on the linear damage mechanics [19], the stress-strain relation for the rock under uniaxial conditions can be expressed as follows:

$$
\varepsilon=\frac{\widetilde{\sigma}}{E}=\frac{\sigma}{E(1-D)},
$$

where $\sigma$ is the stress applied to a damaged material, $\widetilde{\sigma}$ is the stress acting on the undamaged material, $E$ denotes Young's modulus of the undamaged material, and $D$ is a damage variable which takes a value between 0 and 1 corresponding to intact and fully damaged states, respectively. Equation (20) can be rewritten as 


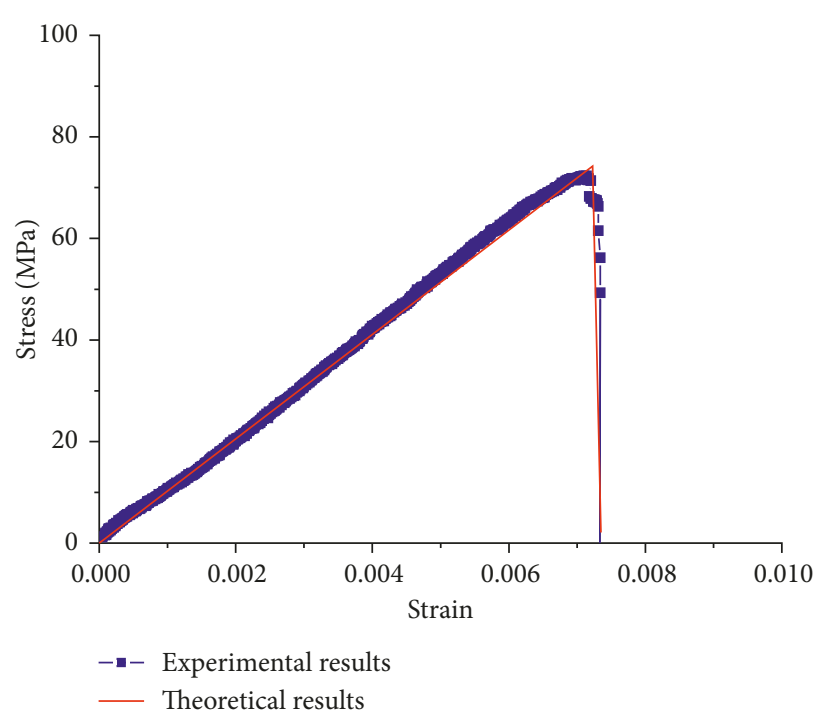

(a)

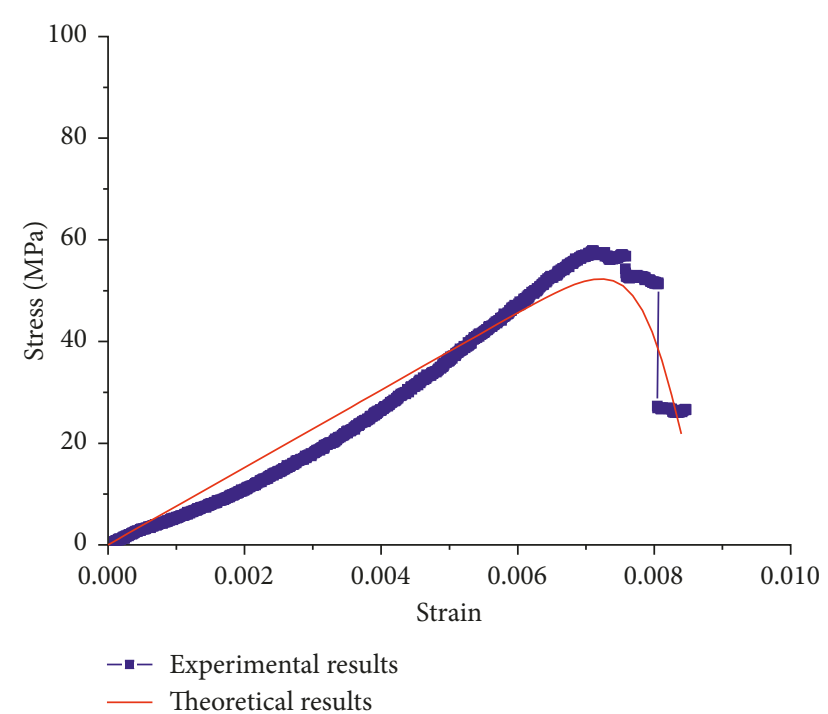

(b)

Figure 6: Comparisons of experimental and theoretical results. (a) Specimen number 10. (b) Specimen number 20.

$$
\sigma=E \varepsilon(1-D)
$$

In probability theory and statistics, let $N$ denote the number of total mesoscopic elements and $N_{t}$ denote the number of failed mesoscopic elements. The damage variable $D$ can be directly defined as

$$
D=\frac{N_{t}(\varepsilon)}{N} .
$$

The Weibull distribution is one of the most important continuous probability distributions. It was first introduced to study the issue of structural strength and life data analysis and has been successfully applied to the mechanical failures of geomaterials [20-22]. In this study, it is confirmed by experiment that the Weibull distribution is also valid. After the strain is regarded as a random variable, the following probability density function is used to describe the mesoscopic element failure behavior:

$$
f(x)=\frac{\beta}{\eta}\left(\frac{\varepsilon-\varepsilon_{0}}{\eta}\right)^{\beta-1} \exp \left[-\left(\frac{\varepsilon-\varepsilon_{0}}{\eta}\right)^{\beta}\right], \quad\left(\varepsilon \geq \varepsilon_{0}\right)
$$

where $\varepsilon_{0}$ is the threshold strain for the damage element; $\varepsilon_{0}=0$ for sandstone in this study; $\eta$ is the mean value; and $\beta$ is the shape parameter.

When the axial strain reaches the value of $\varepsilon$, the number of damaged elements $N_{t}$ may be written as follows:

$$
N_{t}(\varepsilon)=\int_{0}^{\varepsilon} N f(x) d x .
$$

Substituting (23) and (24) into (22), we have

$$
D=\frac{N_{t}(\varepsilon)}{N}=1-\exp \left[-\left(\frac{\varepsilon-\varepsilon_{0}}{\eta}\right)^{\beta}\right] \text {. }
$$

Then, substituting (25) into (21), the following formula can be obtained:

$$
\sigma=E \varepsilon \exp \left[-\left(\frac{\varepsilon-\varepsilon_{0}}{\eta}\right)^{\beta}\right] .
$$

In order to demonstrate the rationality and accuracy of the statistical damage constitutive model proposed in this paper, some typical experimental curves are selected and compared with the theoretical model. The statistical distribution parameters in the constitutive model can be obtained by using the back analysis method. From Figure 6, we find that the theoretical curves almost coincide with the experimental curves, which implies that the statistical damage constitutive model deduced in this paper could well describe the whole stress-strain process of the sandstone rock.

\section{Conclusions}

In this paper, the statistical mechanic behavior of the rock is investigated through a series of uniaxial compression experiments with acoustic emission. The following conclusions can be obtained.

The stress-strain curve can be approximately divided into four typical stages: the compaction stage, the linear elastic stage, the crack propagation stage, and the failure stage. A few events appear suggesting that a little damage of the rock happens in the compaction and elastic stages. As the strain increases to a critical value, the $\mathrm{AE}$ activity increases rapidly, and then, $\mathrm{AE}$ activity decreases with the further increase of strain.

The mechanical parameters were regressed with the Weibull distribution, normal distribution, and log-normal distribution models. The results show that the Weibull distribution can more closely reflect the strength and elastic modulus distribution laws of the rock under uniaxial compression conditions.

A stochastic damage constitutive model for the rock was developed based on the continuous damage and probability 
theories. Comparing theoretical results of the model with experimental data, it is found that the stress-strain relationship could be well described by the damage constitutive model.

\section{Conflicts of Interest}

The authors declare that they have no conflicts of interest.

\section{Acknowledgments}

This research was supported by the State Key Research Development Program of China (2016YFC0600705) and the National Natural Science Foundation of China (51574219 and 51504246).

\section{References}

[1] P. M. Amaral, J. C. Fernandes, and L. G. Rosa, "Weibull statistical analysis of granite bending strength," Rock Mechanics and Rock Engineering, vol. 41, pp. 917-928, 2008.

[2] G. Bruno, G. Vessia, and L. Bobbo, "Statistical method for assessing the uniaxial compressive strength of carbonate rock by Schmidt hammer tests performed on core samples," Rock Mechanics and Rock Engineering, vol. 46, no. 1, pp. 199-206, 2013.

[3] L. Ivanicova, "Statistical evaluation of model rock compressive strength at Branisko exploratory tunnel excavation," Acta Montanistica Slovaca, vol. 9, no. 4, pp. 360-363, 2004.

[4] A. P. Joag and V. S. Lele, "Statistical prediction formula for compressive strength of a rock," Rock Mechanics, vol. 13, no. 4, pp. 215-220, 1981.

[5] E. Hess, D. Bruchman, A. Assakkaf, and M. Ayyub, "Uncertainties in material strength, geometric, and load variables," Naval Engineers Journal, vol. 114, no. 2, pp. 139-166, 2002.

[6] Y. L. Bai, Y. J. Wei, M. F. Xia, and F. J. Ke, "Weibull modulus for diverse strength due to sample-specificity," Theoretical and Applied Fracture Mechanics, vol. 34, no. 3, pp. 211-216, 2000.

[7] A. Azimian, R. Ajalloeian, and L. Fatehi, "An empirical correlation of uniaxial compressive strength with p-wave velocity and point load strength index on marly rocks using statistical method," Geotechnical and Geological Engineering, vol. 32, no. 1, pp. 205-214, 2014.

[8] L. Y. Zhang, "Estimating the strength of jointed rock masses," Rock Mechanics and Rock Engineering, vol. 43, no. 4, pp. 391-402, 2010.

[9] G. B. Lori, "Statistical characterization of meso-scale uniaxial compressive strength in brittle materials with randomly occurring flaws," International Journal of Solids and Structures, vol. 47, no. 18-19, pp. 2398-2413, 2010.

[10] A. Rinaldi and D. Krajcinovic, "Statistical damage mechanics and extreme value theory," International Journal of Damage Mechanics, vol. 16, no. 1, pp. 57-76, 2007.

[11] G. Z. Yin, H. Qin, G. Huang, L. Youchang, and Z. X. Dai, "Acoustic emission from gas-filled coal under triaxial compression," International Journal of Mining Science and Technology, vol. 22, no. 6, pp. 775-778, 2012.

[12] A. Sghafi, A. R. Mirhabibi, and G. H. Yari, "Improved linear regression method for estimating Weibull parameters," Theoretical and Applied Fracture Mechanics, vol. 52, no. 3, pp. 180-182, 2009.
[13] Y. M. Lai, S. Li, J. Qi, Z. Gao, and X. Chang, "Strength distributions of warm frozen clay and its stochastic damage constitutive model," Cold Regions Science and Technology, vol. 53, no. 2, pp. 200-215, 2008.

[14] B. Basu, D. Tiwari, D. Kundu, and R. Prasad, "Is Weibull distribution the most appropriate statistical strength distribution for brittle materials?," Ceramics International, vol. 35, no. 1, pp. 237-246, 2009.

[15] M. Teimouri and A. K. Gupta, "On the three-parameter Weibull distribution shape parameter estimation," Journal of Data Science, vol. 11, pp. 403-414, 2013.

[16] J. Deng and D. S. Gu, "On a statistical damage constitutive model for rock materials," Computer and Geosciences, vol. 37, no. 2, pp. 122-128, 2011.

[17] S. H. Li and D. Zhou, "Progressive failure constitutive model of fracture plane in geomaterial based on strain strength distribution," International Journal of Solids and Structures, vol. 50, no. 3-4, pp. 570-577, 2013.

[18] X. Li, W. G. Cao, and Y. H. Su, "A statistical damage constitutive model for softening behavior of rocks," Engineering Geology, vol. 143-144, pp. 1-17, 2012.

[19] J. Lemaitre and J. L. Chaboche, Mechanics of Solid Materials, Cambridge University Press, Cambridge, UK, 1990.

[20] E. Pena, V. Alastrue, A. Laborda, M. A. Martinez, and M. Doblare, "A constitutive formulation of vascular tissue mechanics including viscoelasticity and softening behavior," Journal of Biomechanics, vol. 43, no. 5, pp. 984-989, 2010.

[21] Y. Wang, Y. C. Chan, Z. L. Gui et al., "Application of Weibull distribution analysis to the dielectric failure of multilayer ceramic capacitors," Materials Science and Engineering B, vol. 47, no. 3, pp. 197-203, 1997.

[22] T. F. Wong, R. Wong, K. T. Chau, and C. A. Tang, "Microcrack statistics, Weibull distribution and micromechanical modeling of compressive failure in rock," Mechanics of Materials, vol. 38, no. 7, pp. 664-681, 2006. 


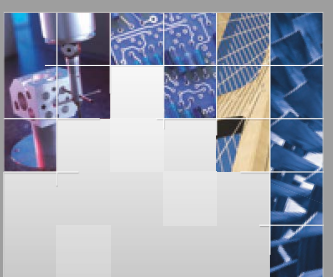

\section{Enfincering}
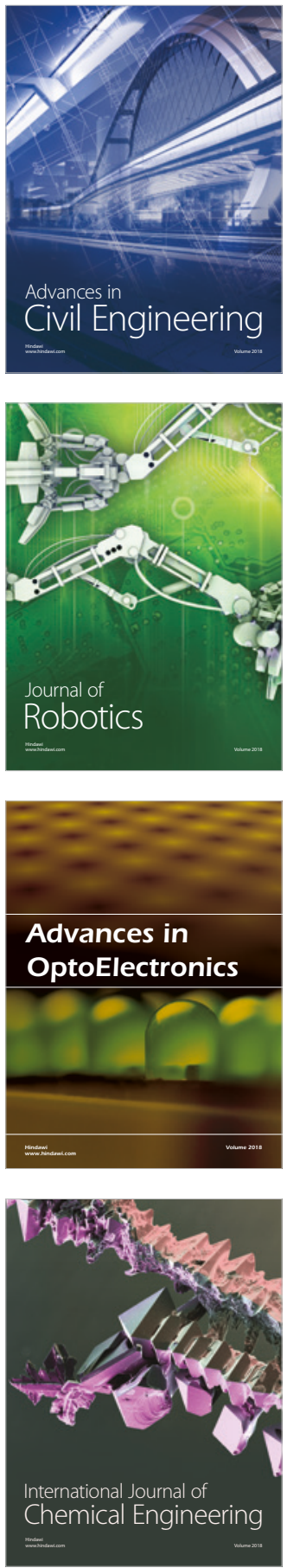

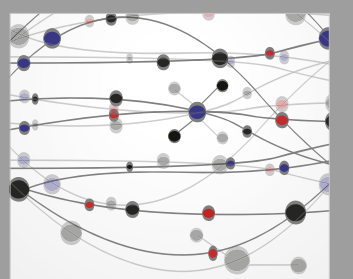

\section{Rotating \\ Machinery}

The Scientific World Journal

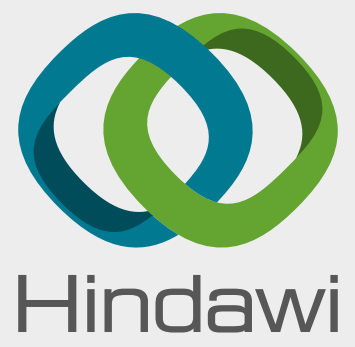

Submit your manuscripts at

www.hindawi.com
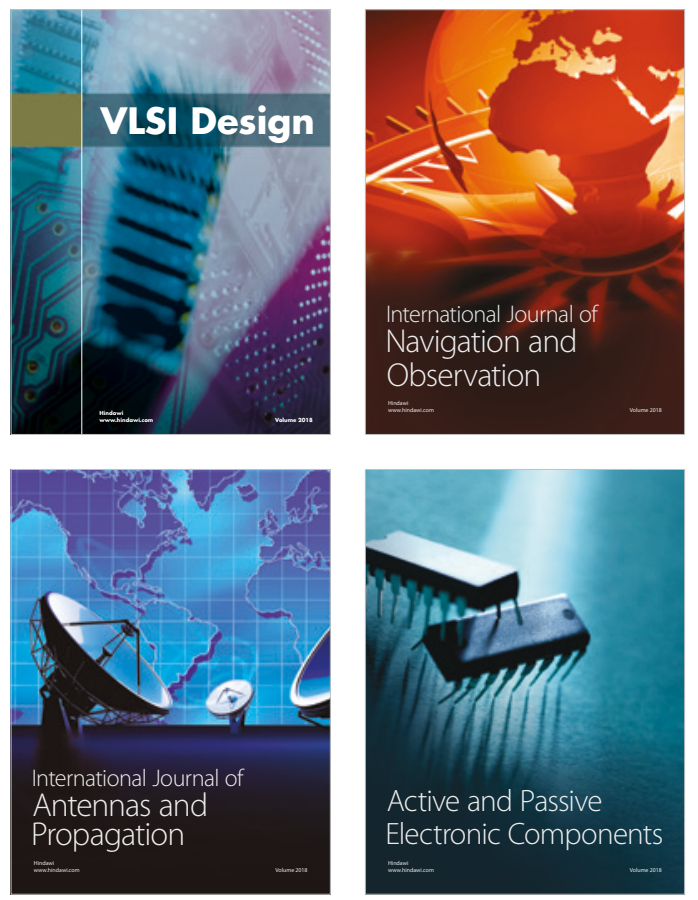
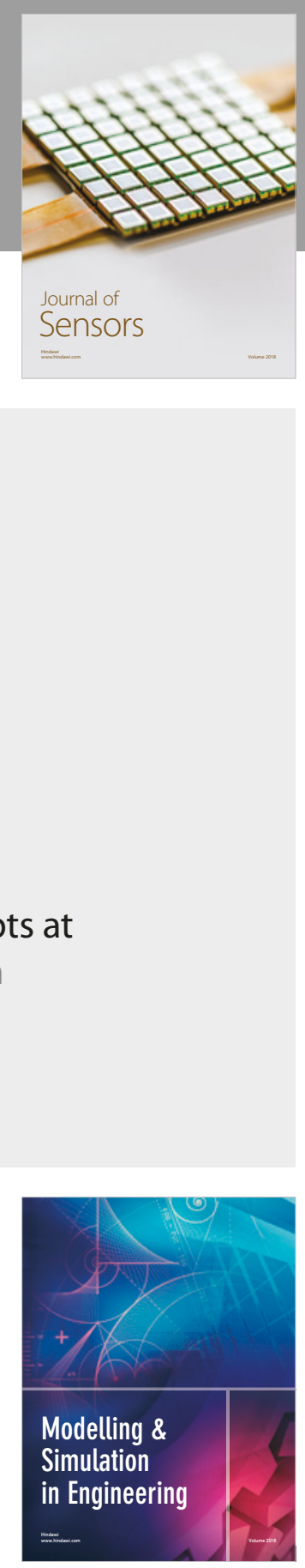

\section{Advances \\ Multimedia}
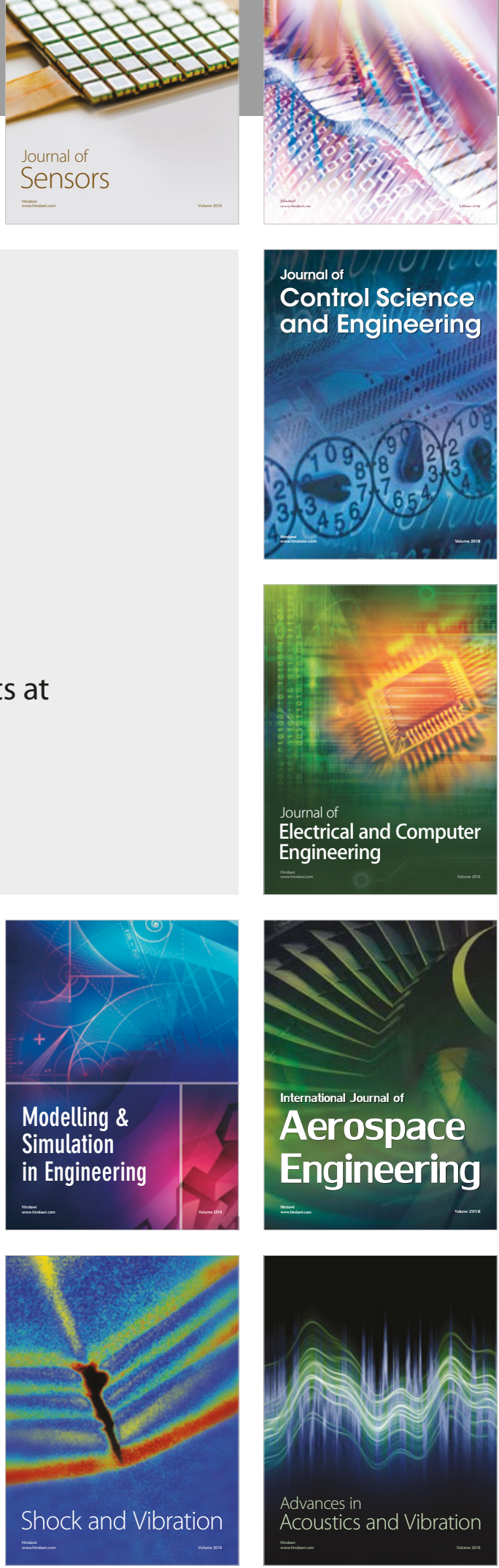